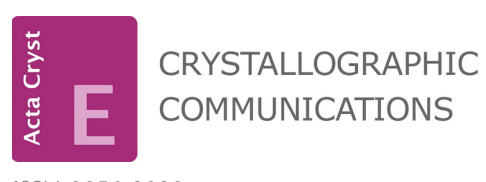

ISSN 2056-9890

Received 8 November 2016

Accepted 26 November 2016

Edited by A. J. Lough, University of Toronto, Canada

Keywords: crystal structure; oxicam; meloxicam; co-crystal; dicarboxylic acid; acetylenedicarboxylic acid; hydrogen bonds; drugs.

CCDC reference: 1506179

Supporting information: this article has supporting information at journals.iucr.org/e
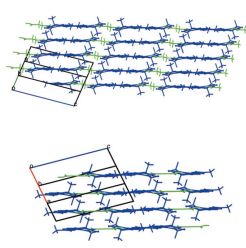

\section{Crystal structure of a 2:1 co-crystal of meloxicam with acetylendicarboxylic acid}

\author{
Christian Tantardini, ${ }^{a *}$ Sergey G. Arkhipov, ${ }^{\mathrm{a}, \mathrm{b} *}$ Ksenya A. Cherkashina, ${ }^{\mathrm{a}, \mathrm{b}}$ \\ Alexander S. Kil'met'ev ${ }^{a, c}$ and Elena V. Boldyreva ${ }^{b *}$
}

aNovosibirsk State University, Pirogova str. 2, Novosibirsk, 630090, Russian Federation, ${ }^{\mathbf{b}}$ Institute of Solid State Chemistry
and Mechanochemistry SB RAS, Kutateladze str. 18, Novosibirsk, 630128, Russian Federation, and ${ }^{\mathbf{c}}$ N. N. Vorozhtsov
Novosibirsk Institute of Organic Chemistry, Lavrentiev str.9, Novosibirsk,630090, Russian Federation. *Correspondence
e-mail: christiantantardini@ymail.com, arksergey@gmail.com, eboldyreva@yahoo.com

The pharmaceutical 2:1 co-crystal of meloxicam [MXM; systematic name: 4-hydroxy-2-methyl- $N$-(5-methylthiazol-2-yl)-2H-1,2-benzothiazine-3-carboxamide 1,1-dioxide] with acetylenedicarboxylic acid (ACA; systematic name: but-2-ynedioic acid), crystallizes with one MXM molecule and half an ACA molecule in the asymmetric unit, $\mathrm{C}_{14} \mathrm{H}_{13} \mathrm{~N}_{3} \mathrm{O}_{4} \mathrm{~S}_{2} \cdot 0.5 \mathrm{C}_{4} \mathrm{H}_{2} \mathrm{O}_{4}$. The mid-point of the triple bond of ACA is located on an inversion centre. In the crystal, the two stereoisomers of MXM with respect to the $\mathrm{N}$ atom of the sulfonamide group are related by the inversion centre. The carbonyl and hydroxy groups belonging to the MXM molecule are involved in an intramolecular $\mathrm{O}-\mathrm{H} \cdots \mathrm{O}$ hydrogen bond. The structure-forming motif includes two MXM molecules linked via an ACA conformer through $\mathrm{N}-\mathrm{H} \cdots \mathrm{O}$ and $\mathrm{O}-\mathrm{H} \cdots \mathrm{N}$ hydrogen bonds, similar to MXM co-crystals with other dicarboxylic acids.

\section{Chemical context}

In recent years, crystal engineering has focused on finding new crystalline forms based on the multi-component crystallization of an active pharmaceutical ingredient (API) with a biologically inactive compound. These complexes are ultimately aimed at being employed in the pharmaceutical industry as tablets, suspensions, powders and any other solid forms for oral administration (Shakhtshneider et al., 2007a,b; Crowley \& Zografi, 2002; Hancock \& Parks, 2000; Shakhtshneider \& Boldyrev, 1993; Willart \& Descamps, 2008; Shakhtshneider et al., 2011; Stephenson et al., 2011). Coformers are typically chosen from among the dicarboxylic acids due to their favourable molecular shape and the presence of functional groups capable of forming multiple hydrogen bonds, combined with their affordability and availability. Meloxicam (MXM), 4-hydroxy-2-methyl- $N$-(5-methyl2-thiazolyl)-2H-1,2-benzothiazine-3-carboxamide-1,1-dioxide, belongs to the oxicam family of APIs and is commonly used in the treatment of rheumatoid arthritis (Myz et al., 2012; Tumanov et al., 2012; Weyna et al. 2012). MXM is known to cocrystallize with numerous aliphatic and aromatic dicarboxylic acids under various conditions (temperature, pressure, solvents). In particular, MXM is known to co-crystallize with dicarboxylic acids of $\mathrm{C}-\mathrm{C}$ bond order 1 (succinic acid) and 2 (fumaric and maleic acids). The aim of this study was to obtain a co-crystal of MXM with a dicarboxylic acid of bond order 3: acetylenedicarboxylic acid (ACA). 
<smiles>Cc1cnc(NC(=O)C2=C(O)c3ccccc3S(=O)(=O)N2C)s1</smiles>

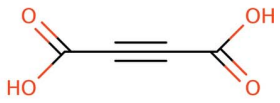

\section{Structural commentary}

The crystal structure of MXM:ACA 2:1 is triclinic with an asymmetric unit that contains one MXM molecule and half of an ACA molecule. The formula unit is generated by an inversion centre which is located at the midpoint of the triple bond of the ACA molecule (Fig. 1). The two stereoisomers of MXM, which differ with respect to the nitrogen atom of the sulfonamide group, are related by an inversion centre in the crystal structure. The dihedral angles between the mean planes of the thiazole and benzene rings of MXM form an almost planar arrangement in terms of the following torsion angles: $\mathrm{S} 2-\mathrm{C} 11-\mathrm{N} 2-\mathrm{H} 2=-174.0^{\circ}, \mathrm{S} 2-\mathrm{C} 11-\mathrm{N} 2-\mathrm{C} 10=$ $6.0(3)^{\circ}, \mathrm{H} 2-\mathrm{N} 2-\mathrm{C} 10-\mathrm{O} 4=176.5^{\circ}, \mathrm{O} 4-\mathrm{C} 10-\mathrm{C} 8-\mathrm{C} 7=$ $10.0(3)^{\circ}, \mathrm{C} 8-\mathrm{C} 7-\mathrm{O} 3-\mathrm{H} 3=-2.2^{\circ}$. The presence of an intramolecular $\mathrm{O}-\mathrm{H} \cdots \mathrm{O}$ hydrogen bond between the carbonyl and hydroxy groups belonging to MXM may account

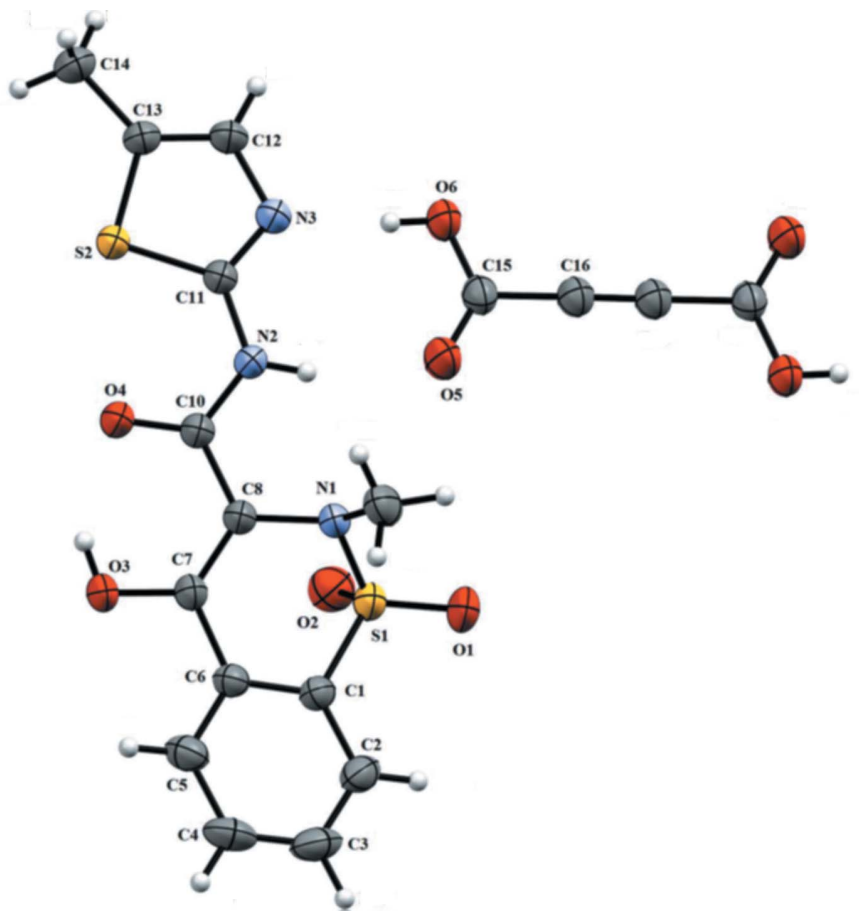

Figure 1

Meloxicam (MXM) and acetylenedicarboxylic acid (ACA) molecules of the 2:1 co-crystal, showing the atom-numbering scheme. Displacement ellipsoids are drawn at the $50 \%$ probability level. Only half of the ACA molecule belongs to the asymmetric unit, as the molecule lies across an inversion centre.
Table 1

Geometrical parameters $\left(\AA{ }^{\circ},{ }^{\circ}\right)$ for the $\mathrm{O}-\mathrm{H} \cdots \mathrm{O}(\mathbf{1}), \mathrm{O}-\mathrm{H} \cdots \mathrm{N}(\mathbf{2})$ and $\mathrm{N}-\mathrm{H} \cdots \mathrm{O}(\mathbf{3})$ interactions in the MXM:ACA 2:1 co-crystal (see also Fig. 3).

\begin{tabular}{lllll}
\hline $\mathrm{D}-\mathrm{H} \cdots \mathrm{A}$ & $\mathrm{D}-\mathrm{H}$ & $\mathrm{H} \cdots \mathrm{A}$ & $\mathrm{D} \cdots \mathrm{A}$ & $\mathrm{D}-\mathrm{H} \cdots \mathrm{A}$ \\
\hline $\mathrm{O} 3-\mathrm{H} 3 \cdots \mathrm{O} 4(\mathbf{1})$ & 0.82 & 1.91 & $2.622(2)$ & 145 \\
$\mathrm{O} 6-\mathrm{H} 6 \cdots \mathrm{N} 3(\mathbf{2})$ & 0.82 & 1.80 & $2.615(3)$ & 174 \\
$\mathrm{~N} 2-\mathrm{H} 2 \cdots \mathrm{O} 5(\mathbf{3})$ & 0.86 & 2.09 & $2.922(3)$ & 164 \\
$\mathrm{O} 3-\mathrm{H} 3 \cdots \mathrm{O} 4{ }^{\mathrm{i}}$ & 0.82 & 2.51 & $2.944(2)$ & 114 \\
\hline
\end{tabular}

Symmetry code: (i) $-x+1,-y+1,-z$.

for the near planarity and the trans position of the $\mathrm{N} 2-\mathrm{H} 2$ group with respect to the carbonyl group $\mathrm{C} 10-\mathrm{O} 4$. The $\mathrm{S} 1 /$ $\mathrm{N} 1 / \mathrm{C} 1 / \mathrm{C} 6 / \mathrm{C} 7 / \mathrm{C} 8 \mathrm{ring}$ is non-planar because of the presence of the sulfonamide group with nitrogen atom N1 in $s p^{3}$ hybridization, with angles $\mathrm{S} 1-\mathrm{N} 1-\mathrm{C} 8=112.79(12)^{\circ}, \mathrm{S} 1-\mathrm{N} 1-\mathrm{C} 9=$ $117.11(14)^{\circ}$ and $\mathrm{C} 9-\mathrm{N} 1-\mathrm{C} 8=115.41(17)^{\circ}$ (bond-angle sum $\left.=345.3^{\circ}\right)$. The overall conformation of this ring is half-chair with atoms $\mathrm{S} 1$ and $\mathrm{N} 1$ being the out-of-plane atoms.

\section{Database survey}

The crystal structures of pure MXM [CCDC ref. code: SEDZOQ (Fabiola et al., 1998)] and its co-crystals with SUCC (MXM-SUCC) (CCDC ref. code: ENICOUM; Cheney et al., 2010) and FUM (MXM-FUM) (CCDC ref. code: ENICIO; Cheney et al., 2010) have the same space group $(P \overline{1})$. The molecular packing in the title compound is shown in Fig. 2. It is similar to that in the crystal structures of pure MXM, as well as of MXM-FUM, MXM-SUCC and MXM-ACA (also shown in Fig. 2). In the co-crystals, some MXM molecules are substituted by the coformer species, maintaining the general packing patterns. The co-crystals MXM-FUM, MXM-SUCC and MXM-ACA have similar structural motifs: two MXM molecules linked by a dicarboxylic acid molecule (Fig. 3).

\section{Supramolecular features}

In the crystal, the components of the structure are linked by $\mathrm{N}-\mathrm{H} \cdots \mathrm{O}$ and $\mathrm{O}-\mathrm{H} \cdots \mathrm{N}$ hydrogen bonds between MXM and ACA, in addition to a long $\mathrm{O}-\mathrm{H} \cdots \mathrm{O}$ interaction, forming chains along [011] which incorporates both $R_{2}^{2}(8)$ and $R_{2}^{2}(12)$ rings. Similar structural motifs have been documented for other MXM co-crystals and in other crystal structures including pure MXM, MXM co-crystals and MXM salts. The structure-forming unit includes two molecules of MXM connected through a dicarboxylic acid molecule acting as a bridge, similar to what has been reported for other MXM cocrystals (Tumanov et al., 2012). Intra- and intermolecular hydrogen bonds are shown in Fig. 3 and their geometrical parameters are summarized in Table 1. The centroid-tocentroid distance between symmetry-related benzene and thiazole rings is 3.7383 (12) $\AA$. These connect the chains into a three-dimensional network. 
(a)

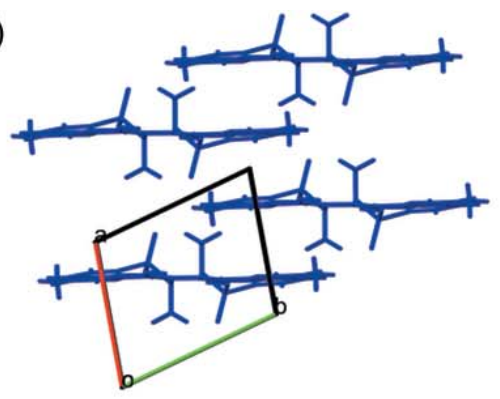

(b)

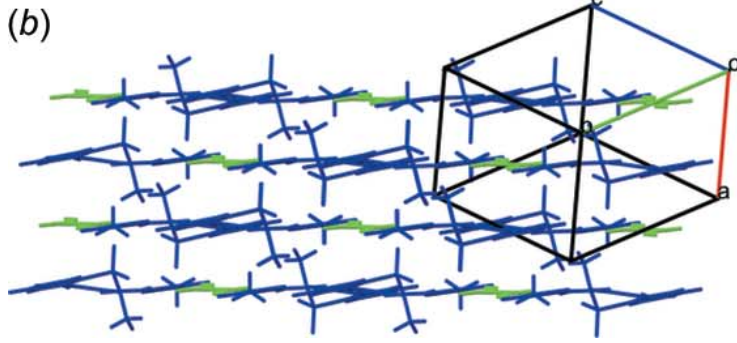

(c)

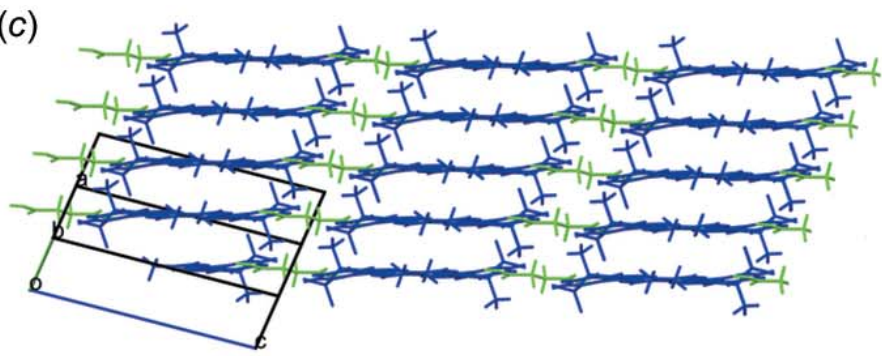

(d)

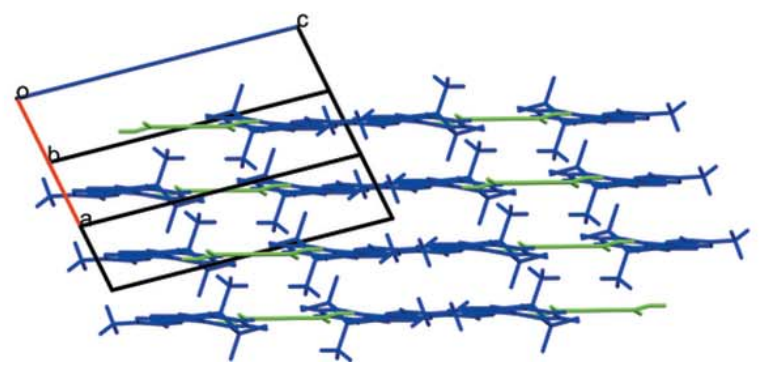

Figure 2

The molecular packing in the crystal structures of $(a)$ pure MXM and its co-crystals $(b)$ MXM-SUCC, $(c)$ MXM-FUM and $(d)$ MXM-ACA.

\section{Synthesis and crystallization}

MXM was purchased from Sigma Aldrich Co Ltd and acetone from Reaktiv. ACA was synthesized through a two-step process from fumaric acid. Fumaric acid was brominated in boiling water (Rhinesmith, 1938) and the resulting 2,3-dibromosuccinic acid was refluxed in potassium hydroxide methanolic solution. ACA was precipitated by adding a concentrated sulfuric acid solution and dried in vacuo (Rhinesmith, 1938). The purity of ACA and the absence of its

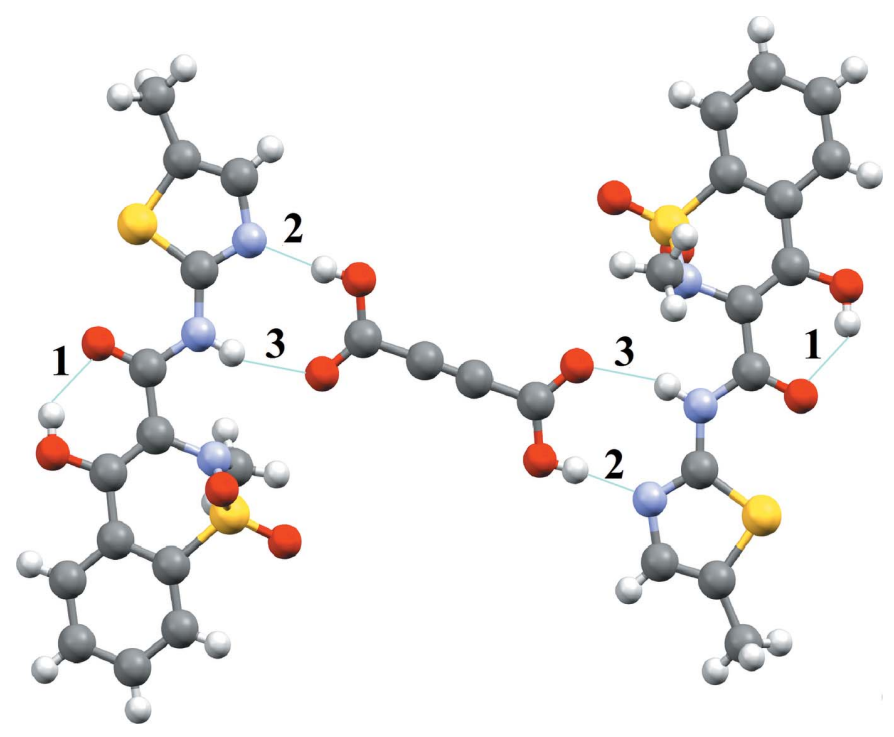

Figure 3

Part of the MXM-ACA 2:1 co-crystal structure showing hydrogen bonds $(\mathbf{1}, \mathbf{2}$ and $\mathbf{3})$ leading to a trimer. The interactions are classified as hydrogen bonds based on the geometric criteria (see text) (Arunan et al., 2011). monohydrate were checked by comparing its experimental powder X-ray diffraction powder (XRPD) pattern with the calculated XRPD patterns of ACA and ACA monohydrate (see S1 in Supporting information). Two polycrystalline samples were obtained by dry and slurry (with acetone) grinding of 1:2 molar mixture of reactants $(0.035 \mathrm{~g}, 0.1 \mathrm{mmol}$ MXM; $0.023 \mathrm{~g}, 0.2 \mathrm{mmol}$ ACA). The 2:1 ratio would correspond to the target stoichiometry and is usually used for obtaining other MXM co-crystals with aliphatic dicarboxylic acids (Myz et al., 2012; Tumanov et al., 2012; Weyna et al. 2012). However, to obtain MXM-ACA 2:1 co-crystals we used a 1:2 MXM:ACA ratio because ACA is highly hygroscopic and converts to its monohydrate form on grinding, not participating then in the co-crystallization. Acetone was used for slurry grinding because it completely dissolves the two starting components (Myz et al., 2012; Tumanov et al., 2012; Weyna et al. 2012). All powder samples were characterized by XRPD using a Stoe Stadi-MP diffractometer with $\mathrm{Cu} K \alpha_{1}$ radiation $(\lambda$ $=1.54060 \AA$ ) at operating potential of $40 \mathrm{kV}$ and electric current of $40 \mathrm{~mA}$, and a Mythen $1 \mathrm{~K}$ detector. All data were processed using WinXPOW (Stoe \& Cie, 1999). Powder diffraction patterns for the samples obtained by grinding and slurry grinding were similar, confirming the possibility to obtain the same product both in the presence and in the absence of a specially added solvent (see S2 in Supporting information); the XRPD patterns of the co-crystal sample were compared with the patterns of the starting reactants, MXM and ACA (see S3 in Supporting information) to prove that a new phase (or a mixture of new phases) had been formed. The ground powder samples were subsequently dissolved in acetone and single crystals were obtained by slow evaporation. Selected crystals were investigated using singlecrystal X-ray diffraction. 
Table 2

Experimental details.

\begin{tabular}{|c|c|}
\hline \multicolumn{2}{|l|}{ Crystal data } \\
\hline Chemical formula & $\mathrm{C}_{14} \mathrm{H}_{13} \mathrm{~N}_{3} \mathrm{O}_{4} \mathrm{~S}_{2} \cdot 0.5 \mathrm{C}_{4} \mathrm{H}_{2} \mathrm{O}_{4}$ \\
\hline$M_{\mathrm{r}}$ & 408.42 \\
\hline Crystal system, space group & Triclinic, $P \overline{1}$ \\
\hline Temperature $(\mathrm{K})$ & 293 \\
\hline$a, b, c(\AA)$ & $7.3861(3), 8.5629(3), 15.1619(6)$ \\
\hline$\alpha, \beta, \gamma\left({ }^{\circ}\right)$ & $75.839(3), 79.196(3), 70.100(3)$ \\
\hline$V\left(\AA^{3}\right)$ & $868.55(6)$ \\
\hline$Z$ & 2 \\
\hline Radiation type & Мо $K \alpha$ \\
\hline$\mu\left(\mathrm{mm}^{-1}\right)$ & 0.35 \\
\hline Crystal size $(\mathrm{mm})$ & $0.4 \times 0.25 \times 0.1$ \\
\hline \multicolumn{2}{|l|}{ Data collection } \\
\hline Diffractometer & $\begin{array}{l}\text { Agilent Xcalibur (Ruby, Gemini } \\
\text { ultra) }\end{array}$ \\
\hline Absorption correction & $\begin{array}{l}\text { Multi-scan (CrysAlis PRO; } \\
\text { Agilent, 2013) }\end{array}$ \\
\hline$T_{\min }, T_{\max }$ & $0.982,1.000$ \\
\hline $\begin{array}{l}\text { No. of measured, independent and } \\
\text { observed }[I>2 \sigma(I)] \text { reflections }\end{array}$ & $10893,3564,2940$ \\
\hline$R_{\text {int }}$ & 0.025 \\
\hline$(\sin \theta / \lambda)_{\max }\left(\AA^{-1}\right)$ & 0.625 \\
\hline \multicolumn{2}{|l|}{ Refinement } \\
\hline$R\left[F^{2}>2 \sigma\left(F^{2}\right)\right], w R\left(F^{2}\right), S$ & $0.036,0.098,1.05$ \\
\hline No. of reflections & 3564 \\
\hline No. of parameters & 248 \\
\hline $\mathrm{H}$-atom treatment & $\mathrm{H}$-atom parameters constrained \\
\hline$\Delta \rho_{\max }, \Delta \rho_{\min }\left(\mathrm{e} \AA^{-3}\right)$ & $0.35,-0.30$ \\
\hline
\end{tabular}

Computer programs: CrysAlis PRO (Agilent, 2013), SHELXS97 (Sheldrick, 2008), SHELXL2014 (Sheldrick, 2015), OLEX2 (Dolomanov et al., 2009), Mercury (Macrae et al., 2008 and publCIF (Westrip, 2010).

\section{Refinement}

Crystal data, data collection and structure refinement details are summarized in Table 2.

All $\mathrm{H}$ atoms were initially located in a difference Fourier map. The positions of all $\mathrm{H}$ atoms were subsequently optimized geometrically and refined using a riding model, with the following assumptions and restraints: $\mathrm{N}-\mathrm{H}=0.86 \AA$ and $U_{\text {iso }}(\mathrm{H})=1.2 U_{\text {eq }}(\mathrm{N})$ for $-\mathrm{N}(\mathrm{H})-$ group, $\mathrm{C}-\mathrm{H}=0.93 \AA$ and $U_{\text {iso }}(\mathrm{H})=1.2 U$ eq $(\mathrm{C})$ for all $\mathrm{C}-\mathrm{H}$ groups, $\mathrm{O}-\mathrm{H}=0.82 \AA$ and $U_{\text {iso }}(\mathrm{H})=1.5 U_{\text {eq }}(\mathrm{O})$ for all $\mathrm{OH}$ groups, $\mathrm{C}-\mathrm{H}=0.96 \AA$ and $U_{\text {iso }}(\mathrm{H})=1.5 U_{\text {eq }}(\mathrm{C})$ for $\mathrm{CH}_{3}$ groups.

For single crystals of MXM:ACA (2:1), two data sets were collected. The first dataset was obtained from a crystal containing four domains, and the second from a single crystal. Unfortunately, the single crystal was very small and at $d_{\mathrm{hkl}} \geq$ $0.80 \AA, R_{\text {int }}$ was $10.2 \%$ and $F^{2} / \sigma\left(F^{2}\right)$ was 3.6. This was significantly worse than the data from the crystal that contained four domains [for the largest domain at $d_{\mathrm{hkl}} \geq 0.80 \AA, R_{\text {int }}$ was $2.50 \%$ and $F^{2} / \sigma\left(F^{2}\right)$ was 28.3$]$. Data obtained from the crystal that contained four domains were processed in three different ways: (1) taking into account the reflections from the largest domain only (one orientation matrix and $74.3 \%$ of all reflections); (2) processing the diffraction data as from multiple crystals (four different orientation matrices) using the hklf5file; (3) processing the diffraction data as from multiple crystals (4 different orientation matrixes) using the. hklf4-file from the largest domain (74.3\% of all reflections). The first and the third processing methods gave approximately the same results, while the first methodology yielded the best results: $R_{\text {int }}=$ 0.025 . This method was therefore chosen for the final structure solution and refinement.

The powder diffraction patterns calculated based on the X-ray single crystal diffraction data were compared with the experimental powder diffraction pattern measured for the sample obtained on grinding, to show that the latter contained a mixture of the MXM:ACA 2:1 co-crystal with some other phases, different from ACA, MXM, or ACA hydrate (see S4 in Supporting information).

\section{Acknowledgements}

This work was supported by the Ministry of Education and Science of the Russian Federation (project No. 1828).

\section{References}

Agilent (2013). CrysAlis PRO, Agilent Technologies, Yarnton, England.

Arunan, E., Desiraju, G. R., Klein, R. A., Sadlej, J., Scheiner, S., Alkorta, I., Clary, D. C., Crabtree, R. H., Dannenberg, J. J., Hobza, P., Kjaergaard, H. G., Legon, A. C., Mennucci, B., Nesbitt, D. J. (2011). Pure Appl. Chem. 83, 1637-1641.

Cheney, M. L., Weyna, D. R., Shan, N., Hanna, M., Wojtas, L. \& Zaworotko, M. J. (2010). Cryst. Growth Des. 10, 4401-4413.

Crowley, K. J. \& Zografi, G. (2002). J. Pharm. Sci. 91, 492-507.

Dolomanov, O. V., Bourhis, L. J., Gildea, R. J., Howard, J. A. K. \& Puschmann, H. (2009). J. Appl. Cryst. 42, 339-341.

Fabiola, G. F., Pattabhi, V., Manjunatha, S. G., Rao, G. V. \& Nagarajan, K. (1998). Acta Cryst. C54, 2001-2003.

Hancock, B. C. \& Parks, M. (2000). Pharm. Res. 17, 397-404.

Macrae, C. F., Bruno, I. J., Chisholm, J. A., Edgington, P. R., McCabe, P., Pidcock, E., Rodriguez-Monge, L., Taylor, R., van de Streek, J. \& Wood, P. A. (2008). J. Appl. Cryst. 41, 466-470.

Myz, S. A., Shakhtshneider, T. P., Tumanov, N. A. \& Boldyreva, E. V. (2012). Russ. Chem. Bull. 61, 1798-1809.

Rhinesmith, H. (1938). Org. Synth. 18, 17.

Shakhtshneider, T. P. \& Boldyrev, V. V. (1993). Drug Dev. Ind. Pharm. 19, 2055-2067.

Shakhtshneider, T. P., Danède, F., Capet, F. J. F., Willart, J. F., Descamps, M., Myz, S. A., Boldyreva, E. V. \& Boldyrev, V. V. (2007b). J. Therm. Anal. Calorim. 89, 699-707.

Shakhtshneider, T. P., Danède, F., Capet, F., Willart, J. F., Descamps, M., Paccou, L., Surov, E. V., Boldyreva, E. V. \& Boldyrev, V. V. (2007a). J. Therm. Anal. Calorim. 89, 709-715.

Shakhtshneider, T. P., Myz, S. A., Dyakonova, M. A., Boldyrev, V. V., Boldyreva, E. V., Nizovskii, A. I., Kalinkin, A. V. \& Kumar, R. (2011). Acta Phys. Pol. A, 120, 272-278.

Sheldrick, G. M. (2008). Acta Cryst. A64, 112-122.

Sheldrick, G. M. (2015). Acta Cryst. C71, 3-8.

Stephenson, G. A., Aburub, A. \& Woods, T. A. (2011). J. Pharm. Sci. 100, 1607-1617.

Stoe \& Cie (1999). WinXPOW. Stoe \& Cie, Darmstadt, Germany.

Tumanov, N. A., Myz, S. A., Shakhtshneider, T. P. \& Boldyreva, E. V. (2012). CrystEngComm, 14, 305-313.

Westrip, S. P. (2010). J. Appl. Cryst. 43, 920-925.

Weyna, D. R., Cheney, M. L., Shan, N., Hanna, M., Zaworotko, M. J., Sava, V., Song, S. \& Sanchez-Ramos, J. R. (2012). Mol. Pharm. 9, 2094-2102.

Willart, J. F. \& Descamps, M. (2008). Mol. Pharm. 5, 905-920. 


\section{supporting information}

Acta Cryst. (2016). E72, 1856-1859 [https://doi.org/10.1107/S2056989016018909]

\section{Crystal structure of a 2:1 co-crystal of meloxicam with acetylendicarboxylic acid}

Christian Tantardini, Sergey G. Arkhipov, Ksenya A. Cherkashina, Alexander S. Kil'met'ev and Elena V. Boldyreva

Computing details

Data collection: CrysAlis PRO (Agilent, 2013); cell refinement: CrysAlis PRO (Agilent, 2013); data reduction: CrysAlis PRO (Agilent, 2013); program(s) used to solve structure: SHELXS97 (Sheldrick, 2008); program(s) used to refine structure: SHELXL2014 (Sheldrick, 2015); molecular graphics: OLEX2 (Dolomanov et al., 2009) and Mercury (Macrae et al., 2008; software used to prepare material for publication: OLEX2 (Dolomanov et al., 2009) and publCIF (Westrip, 2010).

4-Hydroxy-2-methyl-N-(5-methyl-1,3-thiazol-2-yl)-2H-1,2-benzothiazine-3-carboxamide-2-butynedioic acid

Crystal data

$\mathrm{C}_{14} \mathrm{H}_{13} \mathrm{~N}_{3} \mathrm{O}_{4} \mathrm{~S}_{2} \cdot 0.5 \mathrm{C}_{4} \mathrm{H}_{2} \mathrm{O}_{4}$

$M_{r}=408.42$

Triclinic, $P \overline{1}$

$Z=2$

$a=7.3861$ (3) $\AA$

$F(000)=422$

$b=8.5629(3) \AA$

$D_{\mathrm{x}}=1.562 \mathrm{Mg} \mathrm{m}^{-3}$

$c=15.1619(6) \AA$

Mo $K \alpha$ radiation, $\lambda=0.71073 \AA$

$\alpha=75.839(3)^{\circ}$

Cell parameters from 4820 reflections

$\theta=2.6-28.0^{\circ}$

$\beta=79.196(3)^{\circ}$

$\mu=0.35 \mathrm{~mm}^{-1}$

$\gamma=70.100(3)^{\circ}$

$V=868.55(6) \AA^{3}$

$T=293 \mathrm{~K}$

Prism, clear light colourless

$0.4 \times 0.25 \times 0.1 \mathrm{~mm}$

\section{Data collection}

Agilent Xcalibur (Ruby, Gemini ultra) diffractometer

Radiation source: Enhance (Mo) X-ray Source

Graphite monochromator

Detector resolution: 10.3457 pixels $\mathrm{mm}^{-1}$

$\omega$ scans

Absorption correction: multi-scan

(CrysAlis PRO; Agilent, 2013)

$T_{\min }=0.982, T_{\max }=1.000$
10893 measured reflections

3564 independent reflections

2940 reflections with $I>2 \sigma(I)$

$R_{\text {int }}=0.025$

$\theta_{\max }=26.4^{\circ}, \theta_{\min }=2.6^{\circ}$

$h=-9 \rightarrow 9$

$k=-10 \rightarrow 10$

$l=-18 \rightarrow 18$ 


\section{Refinement}

Refinement on $F^{2}$

Least-squares matrix: full

$R\left[F^{2}>2 \sigma\left(F^{2}\right)\right]=0.036$

$w R\left(F^{2}\right)=0.098$

$S=1.05$

3564 reflections

248 parameters

0 restraints
Primary atom site location: structure-invariant direct methods

Hydrogen site location: inferred from neighbouring sites

$\mathrm{H}$-atom parameters constrained

$w=1 /\left[\sigma^{2}\left(F_{\mathrm{o}}^{2}\right)+(0.0463 P)^{2}+0.2383 P\right]$ where $P=\left(F_{\mathrm{o}}{ }^{2}+2 F_{\mathrm{c}}{ }^{2}\right) / 3$

$(\Delta / \sigma)_{\max }<0.001$

$\Delta \rho_{\max }=0.35$ e $\AA^{-3}$

$\Delta \rho_{\min }=-0.30$ e $\AA^{-3}$

Special details

Experimental. Suitable-quality crystals were selected using polarised light under the microscope and mounted by means of MiTiGenMicroGrippers using MiTiGen LV Cryo Oil (LVCO-1) onto an Agilent Xcalibur (Ruby, Gemini Ultra) diffractometer.

Geometry. All esds (except the esd in the dihedral angle between two 1.s. planes) are estimated using the full covariance matrix. The cell esds are taken into account individually in the estimation of esds in distances, angles and torsion angles; correlations between esds in cell parameters are only used when they are defined by crystal symmetry. An approximate (isotropic) treatment of cell esds is used for estimating esds involving 1.s. planes.

Fractional atomic coordinates and isotropic or equivalent isotropic displacement parameters $\left(\hat{A}^{2}\right)$

\begin{tabular}{|c|c|c|c|c|}
\hline & $x$ & $y$ & $z$ & $U_{\text {iso }} * / U_{\text {eq }}$ \\
\hline S2 & $0.26125(7)$ & $0.95843(6)$ & $0.03447(3)$ & $0.03606(14)$ \\
\hline S1 & $0.83244(8)$ & $0.38866(6)$ & $0.33477(3)$ & $0.04072(15)$ \\
\hline $\mathrm{O} 4$ & $0.4415(2)$ & 0.61781 (17) & $0.08344(9)$ & 0.0447 (4) \\
\hline $\mathrm{O} 3$ & $0.6628(2)$ & $0.30130(18)$ & $0.10423(10)$ & 0.0463 (4) \\
\hline H3 & 0.591499 & 0.389131 & 0.077100 & $0.069 *$ \\
\hline O6 & $0.3828(2)$ & $1.10848(17)$ & $0.32189(10)$ & 0.0439 (4) \\
\hline H6 & 0.367153 & 1.088861 & 0.274023 & $0.066^{*}$ \\
\hline $\mathrm{N} 2$ & $0.4321(2)$ & 0.76888 (19) & 0.18889 (11) & $0.0350(4)$ \\
\hline $\mathrm{H} 2$ & 0.457747 & 0.764077 & 0.242714 & $0.042 *$ \\
\hline N3 & $0.3138(2)$ & $1.06452(19)$ & $0.16821(11)$ & 0.0353 (4) \\
\hline $\mathrm{C} 10$ & $0.4825(3)$ & $0.6209(2)$ & $0.15831(13)$ & 0.0336 (4) \\
\hline C11 & $0.3423(3)$ & $0.9263(2)$ & $0.13860(12)$ & 0.0308 (4) \\
\hline $\mathrm{C} 13$ & $0.1832(3)$ & $1.1746(2)$ & $0.03060(13)$ & 0.0339 (4) \\
\hline N1 & $0.6110(2)$ & $0.48326(19)$ & $0.30707(10)$ & 0.0375 (4) \\
\hline $\mathrm{O} 2$ & $0.9585(2)$ & $0.46318(19)$ & $0.26770(11)$ & 0.0517 (4) \\
\hline $\mathrm{C} 12$ & $0.2232(3)$ & $1.2058(2)$ & $0.10662(13)$ & $0.0372(4)$ \\
\hline H12 & 0.191841 & 1.315071 & 0.116962 & $0.045^{*}$ \\
\hline $\mathrm{C} 8$ & $0.5911(3)$ & $0.4690(2)$ & $0.21752(12)$ & 0.0334 (4) \\
\hline C6 & $0.7971(3)$ & $0.1699(2)$ & $0.24281(13)$ & 0.0320 (4) \\
\hline O5 & $0.4837(2)$ & $0.82648(18)$ & $0.36346(10)$ & 0.0529 (4) \\
\hline $\mathrm{C} 7$ & $0.6775(3)$ & $0.3215(2)$ & $0.18691(13)$ & $0.0331(4)$ \\
\hline $\mathrm{C} 1$ & $0.8718(3)$ & $0.1834(2)$ & $0.31771(13)$ & 0.0337 (4) \\
\hline $\mathrm{C} 15$ & $0.4507(3)$ & $0.9670(2)$ & $0.37751(13)$ & 0.0355 (4) \\
\hline $\mathrm{C} 16$ & $0.4862(3)$ & $0.9913(2)$ & $0.46450(13)$ & 0.0379 (4) \\
\hline $\mathrm{O} 1$ & $0.8318(3)$ & $0.3844(2)$ & $0.42935(10)$ & $0.0621(5)$ \\
\hline $\mathrm{C} 2$ & $0.9717(3)$ & 0.0418 & $0.37611(14)$ & $0.0423(5)$ \\
\hline
\end{tabular}




$\begin{array}{lllll}\text { H2A } & 1.018860 & 0.053179 & 0.426149 & 0.051^{*} \\ \text { C5 } & 0.8338(3) & 0.0088(2) & 0.22541(16) & 0.0419(5) \\ \text { H5 } & 0.791005 & -0.003525 & 0.174357 & 0.050^{*} \\ \text { C14 } & 0.0890(3) & 1.2967(3) & -0.04937(14) & 0.0443(5) \\ \text { H14A } & 0.153415 & 1.258732 & -0.104979 & 0.066^{*} \\ \text { H14B } & 0.098095 & 1.406802 & -0.051516 & 0.066^{*} \\ \text { H14C } & -0.044963 & 1.302810 & -0.042813 & 0.066^{*} \\ \text { C3 } & 1.0008(3) & -0.1169(3) & 0.35939(16) & 0.0506(6) \\ \text { H3A } & 1.065483 & -0.213032 & 0.399023 & 0.061^{*} \\ \text { C4 } & 0.9342(3) & -0.1333(3) & 0.28424(17) & 0.0492(6) \\ \text { H4 } & 0.956697 & -0.240531 & 0.272748 & 0.059^{*} \\ \text { C9 } & 0.4499(3) & 0.4608(3) & 0.37857(15) & 0.0503(6) \\ \text { H9A } & 0.456712 & 0.343501 & 0.394250 & 0.075^{*} \\ \text { H9B } & 0.328377 & 0.527269 & 0.355785 & 0.075^{*} \\ \text { H9C } & 0.460436 & 0.497153 & 0.431969 & 0.075^{*}\end{array}$

Atomic displacement parameters $\left(\AA^{2}\right)$

\begin{tabular}{lllllll}
\hline & $U^{11}$ & $U^{22}$ & $U^{33}$ & $U^{12}$ & $U^{13}$ & $U^{23}$ \\
\hline S2 & $0.0495(3)$ & $0.0272(3)$ & $0.0303(3)$ & $-0.0048(2)$ & $-0.0144(2)$ & $-0.00641(19)$ \\
S1 & $0.0582(3)$ & $0.0324(3)$ & $0.0323(3)$ & $-0.0072(2)$ & $-0.0181(2)$ & $-0.0080(2)$ \\
O4 & $0.0633(9)$ & $0.0325(7)$ & $0.0356(8)$ & $-0.0010(6)$ & $-0.0228(7)$ & $-0.0089(6)$ \\
O3 & $0.0660(10)$ & $0.0363(8)$ & $0.0350(8)$ & $0.0000(7)$ & $-0.0215(7)$ & $-0.0148(6)$ \\
O6 & $0.0624(9)$ & $0.0372(8)$ & $0.0364(8)$ & $-0.0121(7)$ & $-0.0180(7)$ & $-0.0106(6)$ \\
N2 & $0.0472(9)$ & $0.0265(8)$ & $0.0282(8)$ & $-0.0024(7)$ & $-0.0135(7)$ & $-0.0055(6)$ \\
N3 & $0.0457(9)$ & $0.0275(8)$ & $0.0322(9)$ & $-0.0066(7)$ & $-0.0112(7)$ & $-0.0066(7)$ \\
C10 & $0.0408(10)$ & $0.0284(10)$ & $0.0299(10)$ & $-0.0046(8)$ & $-0.0096(8)$ & $-0.0071(8)$ \\
C11 & $0.0357(10)$ & $0.0264(9)$ & $0.0285(10)$ & $-0.0046(7)$ & $-0.0073(8)$ & $-0.0067(7)$ \\
C13 & $0.0396(10)$ & $0.0265(9)$ & $0.0321(10)$ & $-0.0052(8)$ & $-0.0080(8)$ & $-0.0036(8)$ \\
N1 & $0.0532(10)$ & $0.0279(8)$ & $0.0258(8)$ & $-0.0002(7)$ & $-0.0103(7)$ & $-0.0086(6)$ \\
O2 & $0.0613(10)$ & $0.0429(9)$ & $0.0583(10)$ & $-0.0217(7)$ & $-0.0158(8)$ & $-0.0085(7)$ \\
C12 & $0.0489(11)$ & $0.0239(9)$ & $0.0365(11)$ & $-0.0056(8)$ & $-0.0104(9)$ & $-0.0054(8)$ \\
C8 & $0.0432(10)$ & $0.0270(9)$ & $0.0275(10)$ & $-0.0040(8)$ & $-0.0097(8)$ & $-0.0065(7)$ \\
C6 & $0.0355(10)$ & $0.0252(9)$ & $0.0323(10)$ & $-0.0057(7)$ & $-0.0031(8)$ & $-0.0060(7)$ \\
O5 & $0.0811(11)$ & $0.0372(9)$ & $0.0457(9)$ & $-0.0123(8)$ & $-0.0245(8)$ & $-0.0130(7)$ \\
C7 & $0.0420(10)$ & $0.0295(10)$ & $0.0289(10)$ & $-0.0082(8)$ & $-0.0095(8)$ & $-0.0075(7)$ \\
C1 & $0.0382(10)$ & $0.0279(10)$ & $0.0296(10)$ & $-0.0049(8)$ & $-0.0042(8)$ & $-0.0035(7)$ \\
C15 & $0.0373(10)$ & $0.0382(11)$ & $0.0335(11)$ & $-0.0104(8)$ & $-0.0060(8)$ & $-0.0117(8)$ \\
C16 & $0.0454(11)$ & $0.0360(11)$ & $0.0353(10)$ & $-0.0126(9)$ & $-0.0092(9)$ & $-0.0085(9)$ \\
O1 & $0.0908(13)$ & $0.0541(10)$ & $0.0382(9)$ & $-0.0004(9)$ & $-0.0318(8)$ & $-0.0160(7)$ \\
C2 & $0.0427(11)$ & $0.0398(12)$ & $0.0330(11)$ & $-0.0022(9)$ & $-0.0064(9)$ & $0.0002(9)$ \\
C5 & $0.0437(11)$ & $0.0300(10)$ & $0.0509(13)$ & $-0.0066(8)$ & $-0.0056(9)$ & $-0.0131(9)$ \\
C14 & $0.0556(13)$ & $0.0324(11)$ & $0.0392(12)$ & $-0.0047(9)$ & $-0.0152(10)$ & $-0.0020(9)$ \\
C3 & $0.0471(12)$ & $0.0329(11)$ & $0.0521(14)$ & $0.0017(9)$ & $-0.0045(10)$ & $0.0064(10)$ \\
C4 & $0.0489(12)$ & $0.0241(10)$ & $0.0668(16)$ & $-0.0041(9)$ & $-0.0028(11)$ & $-0.0078(10)$ \\
C9 & $0.0668(15)$ & $0.0407(12)$ & $0.0338(12)$ & $-0.0061(10)$ & $0.0005(10)$ & $-0.0099(9)$ \\
& & & & & & \\
\hline & & & & & & \\
\hline
\end{tabular}


Geometric parameters $\left(\AA,{ }^{\circ}\right)$

\begin{tabular}{|c|c|c|c|}
\hline $\mathrm{S} 2-\mathrm{C} 11$ & $1.7196(18)$ & $\mathrm{C} 8-\mathrm{C} 7$ & $1.359(2)$ \\
\hline $\mathrm{S} 2-\mathrm{C} 13$ & $1.7306(18)$ & $\mathrm{C} 6-\mathrm{C} 7$ & $1.464(3)$ \\
\hline $\mathrm{S} 1-\mathrm{N} 1$ & $1.6422(17)$ & $\mathrm{C} 6-\mathrm{C} 1$ & $1.397(3)$ \\
\hline $\mathrm{S} 1-\mathrm{O} 2$ & $1.4284(16)$ & $\mathrm{C} 6-\mathrm{C} 5$ & $1.394(3)$ \\
\hline $\mathrm{S} 1-\mathrm{C} 1$ & $1.7567(19)$ & $\mathrm{O} 5-\mathrm{C} 15$ & $1.208(2)$ \\
\hline $\mathrm{S} 1-\mathrm{O} 1$ & $1.4246(15)$ & $\mathrm{C} 1-\mathrm{C} 2$ & $1.382(3)$ \\
\hline $\mathrm{O} 4-\mathrm{C} 10$ & $1.237(2)$ & $\mathrm{C} 15-\mathrm{C} 16$ & $1.467(3)$ \\
\hline $\mathrm{O} 3-\mathrm{H} 3$ & 0.8200 & $\mathrm{C} 16-\mathrm{C} 16^{\mathrm{i}}$ & $1.185(4)$ \\
\hline $\mathrm{O} 3-\mathrm{C} 7$ & $1.335(2)$ & $\mathrm{C} 2-\mathrm{H} 2 \mathrm{~A}$ & 0.9300 \\
\hline O6- $\mathrm{H} 6$ & 0.8200 & $\mathrm{C} 2-\mathrm{C} 3$ & $1.381(3)$ \\
\hline $\mathrm{O} 6-\mathrm{C} 15$ & $1.294(2)$ & $\mathrm{C} 5-\mathrm{H} 5$ & 0.9300 \\
\hline $\mathrm{N} 2-\mathrm{H} 2$ & 0.8600 & $\mathrm{C} 5-\mathrm{C} 4$ & $1.389(3)$ \\
\hline $\mathrm{N} 2-\mathrm{C} 10$ & $1.364(2)$ & $\mathrm{C} 14-\mathrm{H} 14 \mathrm{~A}$ & 0.9600 \\
\hline $\mathrm{N} 2-\mathrm{C} 11$ & $1.384(2)$ & $\mathrm{C} 14-\mathrm{H} 14 \mathrm{~B}$ & 0.9600 \\
\hline N3-C11 & $1.304(2)$ & $\mathrm{C} 14-\mathrm{H} 14 \mathrm{C}$ & 0.9600 \\
\hline $\mathrm{N} 3-\mathrm{C} 12$ & $1.383(2)$ & $\mathrm{C} 3-\mathrm{H} 3 \mathrm{~A}$ & 0.9300 \\
\hline $\mathrm{C} 10-\mathrm{C} 8$ & $1.459(2)$ & $\mathrm{C} 3-\mathrm{C} 4$ & $1.376(3)$ \\
\hline $\mathrm{C} 13-\mathrm{C} 12$ & $1.349(3)$ & $\mathrm{C} 4-\mathrm{H} 4$ & 0.9300 \\
\hline $\mathrm{C} 13-\mathrm{C} 14$ & $1.500(3)$ & $\mathrm{C} 9-\mathrm{H} 9 \mathrm{~A}$ & 0.9600 \\
\hline $\mathrm{N} 1-\mathrm{C} 8$ & $1.431(2)$ & C9-H9B & 0.9600 \\
\hline $\mathrm{N} 1-\mathrm{C} 9$ & $1.482(3)$ & $\mathrm{C} 9-\mathrm{H} 9 \mathrm{C}$ & 0.9600 \\
\hline $\mathrm{C} 12-\mathrm{H} 12$ & 0.9300 & & \\
\hline $\mathrm{C} 11-\mathrm{S} 2-\mathrm{C} 13$ & $89.50(9)$ & $\mathrm{O} 3-\mathrm{C} 7-\mathrm{C} 8$ & $123.70(17)$ \\
\hline $\mathrm{N} 1-\mathrm{S} 1-\mathrm{C} 1$ & $100.92(9)$ & $\mathrm{O} 3-\mathrm{C} 7-\mathrm{C} 6$ & $114.19(16)$ \\
\hline $\mathrm{O} 2-\mathrm{S} 1-\mathrm{N} 1$ & $107.52(9)$ & $\mathrm{C} 8-\mathrm{C} 7-\mathrm{C} 6$ & $122.11(17)$ \\
\hline $\mathrm{O} 2-\mathrm{S} 1-\mathrm{C} 1$ & $108.23(9)$ & $\mathrm{C} 6-\mathrm{C} 1-\mathrm{S} 1$ & $117.03(14)$ \\
\hline $\mathrm{O} 1-\mathrm{S} 1-\mathrm{N} 1$ & $108.86(9)$ & $\mathrm{C} 2-\mathrm{C} 1-\mathrm{S} 1$ & $121.39(16)$ \\
\hline $\mathrm{O} 1-\mathrm{S} 1-\mathrm{O} 2$ & $119.90(11)$ & $\mathrm{C} 2-\mathrm{C} 1-\mathrm{C} 6$ & $121.57(18)$ \\
\hline $\mathrm{O} 1-\mathrm{S} 1-\mathrm{C} 1$ & $109.73(10)$ & $\mathrm{O} 6-\mathrm{C} 15-\mathrm{C} 16$ & $112.85(17)$ \\
\hline $\mathrm{C} 7-\mathrm{O} 3-\mathrm{H} 3$ & 109.5 & $\mathrm{O} 5-\mathrm{C} 15-\mathrm{O} 6$ & $126.38(18)$ \\
\hline $\mathrm{C} 15-\mathrm{O} 6-\mathrm{H} 6$ & 109.5 & $\mathrm{O} 5-\mathrm{C} 15-\mathrm{C} 16$ & $120.77(18)$ \\
\hline $\mathrm{C} 10-\mathrm{N} 2-\mathrm{H} 2$ & 118.0 & $\mathrm{C} 16-\mathrm{C} 16-\mathrm{C} 15$ & $178.9(3)$ \\
\hline $\mathrm{C} 10-\mathrm{N} 2-\mathrm{C} 11$ & $124.02(16)$ & $\mathrm{C} 1-\mathrm{C} 2-\mathrm{H} 2 \mathrm{~A}$ & 120.4 \\
\hline $\mathrm{C} 11-\mathrm{N} 2-\mathrm{H} 2$ & 118.0 & $\mathrm{C} 3-\mathrm{C} 2-\mathrm{C} 1$ & $119.3(2)$ \\
\hline $\mathrm{C} 11-\mathrm{N} 3-\mathrm{C} 12$ & $110.79(16)$ & $\mathrm{C} 3-\mathrm{C} 2-\mathrm{H} 2 \mathrm{~A}$ & 120.4 \\
\hline $\mathrm{O} 4-\mathrm{C} 10-\mathrm{N} 2$ & $121.31(16)$ & $\mathrm{C} 6-\mathrm{C} 5-\mathrm{H} 5$ & 120.0 \\
\hline $\mathrm{O} 4-\mathrm{C} 10-\mathrm{C} 8$ & $122.38(16)$ & $\mathrm{C} 4-\mathrm{C} 5-\mathrm{C} 6$ & $120.1(2)$ \\
\hline $\mathrm{N} 2-\mathrm{C} 10-\mathrm{C} 8$ & $116.29(16)$ & $\mathrm{C} 4-\mathrm{C} 5-\mathrm{H} 5$ & 120.0 \\
\hline $\mathrm{N} 2-\mathrm{C} 11-\mathrm{S} 2$ & $124.41(13)$ & $\mathrm{C} 13-\mathrm{C} 14-\mathrm{H} 14 \mathrm{~A}$ & 109.5 \\
\hline $\mathrm{N} 3-\mathrm{C} 11-\mathrm{S} 2$ & $114.56(13)$ & $\mathrm{C} 13-\mathrm{C} 14-\mathrm{H} 14 \mathrm{~B}$ & 109.5 \\
\hline $\mathrm{N} 3-\mathrm{C} 11-\mathrm{N} 2$ & $121.03(16)$ & $\mathrm{C} 13-\mathrm{C} 14-\mathrm{H} 14 \mathrm{C}$ & 109.5 \\
\hline $\mathrm{C} 12-\mathrm{C} 13-\mathrm{S} 2$ & $109.53(14)$ & $\mathrm{H} 14 \mathrm{~A}-\mathrm{C} 14-\mathrm{H} 14 \mathrm{~B}$ & 109.5 \\
\hline $\mathrm{C} 12-\mathrm{C} 13-\mathrm{C} 14$ & $129.41(17)$ & $\mathrm{H} 14 \mathrm{~A}-\mathrm{C} 14-\mathrm{H} 14 \mathrm{C}$ & 109.5 \\
\hline $\mathrm{C} 14-\mathrm{C} 13-\mathrm{S} 2$ & $121.06(14)$ & $\mathrm{H} 14 \mathrm{~B}-\mathrm{C} 14-\mathrm{H} 14 \mathrm{C}$ & 109.5 \\
\hline $\mathrm{C} 8-\mathrm{N} 1-\mathrm{S} 1$ & $112.79(12)$ & $\mathrm{C} 2-\mathrm{C} 3-\mathrm{H} 3 \mathrm{~A}$ & 119.9 \\
\hline
\end{tabular}




\begin{tabular}{|c|c|c|c|}
\hline $\mathrm{C} 8-\mathrm{N} 1-\mathrm{C} 9$ & $115.41(17)$ & $\mathrm{C} 4-\mathrm{C} 3-\mathrm{C} 2$ & $120.20(19)$ \\
\hline $\mathrm{C} 9-\mathrm{N} 1-\mathrm{S} 1$ & $117.11(14)$ & $\mathrm{C} 4-\mathrm{C} 3-\mathrm{H} 3 \mathrm{~A}$ & 119.9 \\
\hline $\mathrm{N} 3-\mathrm{C} 12-\mathrm{H} 12$ & 122.2 & $\mathrm{C} 5-\mathrm{C} 4-\mathrm{H} 4$ & 119.7 \\
\hline $\mathrm{C} 13-\mathrm{C} 12-\mathrm{N} 3$ & $115.61(17)$ & $\mathrm{C} 3-\mathrm{C} 4-\mathrm{C} 5$ & $120.6(2)$ \\
\hline $\mathrm{C} 13-\mathrm{C} 12-\mathrm{H} 12$ & 122.2 & $\mathrm{C} 3-\mathrm{C} 4-\mathrm{H} 4$ & 119.7 \\
\hline $\mathrm{N} 1-\mathrm{C} 8-\mathrm{C} 10$ & $117.67(15)$ & $\mathrm{N} 1-\mathrm{C} 9-\mathrm{H} 9 \mathrm{~A}$ & 109.5 \\
\hline $\mathrm{C} 7-\mathrm{C} 8-\mathrm{C} 10$ & $120.92(16)$ & $\mathrm{N} 1-\mathrm{C} 9-\mathrm{H} 9 \mathrm{~B}$ & 109.5 \\
\hline $\mathrm{C} 7-\mathrm{C} 8-\mathrm{N} 1$ & $121.34(16)$ & $\mathrm{N} 1-\mathrm{C} 9-\mathrm{H} 9 \mathrm{C}$ & 109.5 \\
\hline $\mathrm{C} 1-\mathrm{C} 6-\mathrm{C} 7$ & $120.26(16)$ & $\mathrm{H} 9 \mathrm{~A}-\mathrm{C} 9-\mathrm{H} 9 \mathrm{~B}$ & 109.5 \\
\hline $\mathrm{C} 5-\mathrm{C} 6-\mathrm{C} 7$ & $121.56(18)$ & $\mathrm{H} 9 \mathrm{~A}-\mathrm{C} 9-\mathrm{H} 9 \mathrm{C}$ & 109.5 \\
\hline $\mathrm{C} 5-\mathrm{C} 6-\mathrm{C} 1$ & $118.14(17)$ & $\mathrm{H} 9 \mathrm{~B}-\mathrm{C} 9-\mathrm{H} 9 \mathrm{C}$ & 109.5 \\
\hline $\mathrm{S} 2-\mathrm{C} 13-\mathrm{C} 12-\mathrm{N} 3$ & $0.0(2)$ & $\mathrm{O} 2-\mathrm{S} 1-\mathrm{C} 1-\mathrm{C} 2$ & $105.81(18)$ \\
\hline $\mathrm{S} 1-\mathrm{N} 1-\mathrm{C} 8-\mathrm{C} 10$ & $-135.40(16)$ & $\mathrm{C} 12-\mathrm{N} 3-\mathrm{C} 11-\mathrm{S} 2$ & $-0.1(2)$ \\
\hline $\mathrm{S} 1-\mathrm{N} 1-\mathrm{C} 8-\mathrm{C} 7$ & $41.7(2)$ & $\mathrm{C} 12-\mathrm{N} 3-\mathrm{C} 11-\mathrm{N} 2$ & $179.66(17)$ \\
\hline $\mathrm{S} 1-\mathrm{C} 1-\mathrm{C} 2-\mathrm{C} 3$ & $-179.35(15)$ & $\mathrm{C} 6-\mathrm{C} 1-\mathrm{C} 2-\mathrm{C} 3$ & $0.9(3)$ \\
\hline $\mathrm{O} 4-\mathrm{C} 10-\mathrm{C} 8-\mathrm{N} 1$ & $-172.87(18)$ & $\mathrm{C} 6-\mathrm{C} 5-\mathrm{C} 4-\mathrm{C} 3$ & $-0.9(3)$ \\
\hline $\mathrm{O} 4-\mathrm{C} 10-\mathrm{C} 8-\mathrm{C} 7$ & $10.0(3)$ & $\mathrm{C} 7-\mathrm{C} 6-\mathrm{C} 1-\mathrm{S} 1$ & $-5.1(2)$ \\
\hline $\mathrm{N} 2-\mathrm{C} 10-\mathrm{C} 8-\mathrm{N} 1$ & $8.8(3)$ & $\mathrm{C} 7-\mathrm{C} 6-\mathrm{C} 1-\mathrm{C} 2$ & $174.63(18)$ \\
\hline $\mathrm{N} 2-\mathrm{C} 10-\mathrm{C} 8-\mathrm{C} 7$ & $-168.30(18)$ & $\mathrm{C} 7-\mathrm{C} 6-\mathrm{C} 5-\mathrm{C} 4$ & $-174.64(18)$ \\
\hline $\mathrm{C} 10-\mathrm{N} 2-\mathrm{C} 11-\mathrm{S} 2$ & $6.0(3)$ & $\mathrm{C} 1-\mathrm{S} 1-\mathrm{N} 1-\mathrm{C} 8$ & $-54.93(15)$ \\
\hline $\mathrm{C} 10-\mathrm{N} 2-\mathrm{C} 11-\mathrm{N} 3$ & $-173.71(17)$ & $\mathrm{C} 1-\mathrm{S} 1-\mathrm{N} 1-\mathrm{C} 9$ & $82.64(15)$ \\
\hline $\mathrm{C} 10-\mathrm{C} 8-\mathrm{C} 7-\mathrm{O} 3$ & $-3.3(3)$ & $\mathrm{C} 1-\mathrm{C} 6-\mathrm{C} 7-\mathrm{O} 3$ & $161.27(17)$ \\
\hline $\mathrm{C} 10-\mathrm{C} 8-\mathrm{C} 7-\mathrm{C} 6$ & $176.22(18)$ & $\mathrm{C} 1-\mathrm{C} 6-\mathrm{C} 7-\mathrm{C} 8$ & $-18.3(3)$ \\
\hline $\mathrm{C} 11-\mathrm{S} 2-\mathrm{C} 13-\mathrm{C} 12$ & $-0.05(15)$ & $\mathrm{C} 1-\mathrm{C} 6-\mathrm{C} 5-\mathrm{C} 4$ & $3.0(3)$ \\
\hline $\mathrm{C} 11-\mathrm{S} 2-\mathrm{C} 13-\mathrm{C} 14$ & $179.40(17)$ & $\mathrm{C} 1-\mathrm{C} 2-\mathrm{C} 3-\mathrm{C} 4$ & $1.3(3)$ \\
\hline $\mathrm{C} 11-\mathrm{N} 2-\mathrm{C} 10-\mathrm{O} 4$ & $-3.5(3)$ & $\mathrm{O} 1-\mathrm{S} 1-\mathrm{N} 1-\mathrm{C} 8$ & $-170.34(14)$ \\
\hline $\mathrm{C} 11-\mathrm{N} 2-\mathrm{C} 10-\mathrm{C} 8$ & $174.84(17)$ & $\mathrm{O} 1-\mathrm{S} 1-\mathrm{N} 1-\mathrm{C} 9$ & $-32.77(17)$ \\
\hline $\mathrm{C} 11-\mathrm{N} 3-\mathrm{C} 12-\mathrm{C} 13$ & $0.0(3)$ & $\mathrm{O} 1-\mathrm{S} 1-\mathrm{C} 1-\mathrm{C} 6$ & $153.05(15)$ \\
\hline $\mathrm{C} 13-\mathrm{S} 2-\mathrm{C} 11-\mathrm{N} 2$ & $-179.64(17)$ & $\mathrm{O} 1-\mathrm{S} 1-\mathrm{C} 1-\mathrm{C} 2$ & $-26.7(2)$ \\
\hline $\mathrm{C} 13-\mathrm{S} 2-\mathrm{C} 11-\mathrm{N} 3$ & $0.07(15)$ & $\mathrm{C} 2-\mathrm{C} 3-\mathrm{C} 4-\mathrm{C} 5$ & $-1.3(3)$ \\
\hline $\mathrm{N} 1-\mathrm{S} 1-\mathrm{C} 1-\mathrm{C} 6$ & $38.29(16)$ & $\mathrm{C} 5-\mathrm{C} 6-\mathrm{C} 7-\mathrm{O} 3$ & $-21.1(3)$ \\
\hline $\mathrm{N} 1-\mathrm{S} 1-\mathrm{C} 1-\mathrm{C} 2$ & $-141.46(17)$ & $\mathrm{C} 5-\mathrm{C} 6-\mathrm{C} 7-\mathrm{C} 8$ & $159.28(19)$ \\
\hline $\mathrm{N} 1-\mathrm{C} 8-\mathrm{C} 7-\mathrm{O} 3$ & $179.69(18)$ & $\mathrm{C} 5-\mathrm{C} 6-\mathrm{C} 1-\mathrm{S} 1$ & $177.20(15)$ \\
\hline $\mathrm{N} 1-\mathrm{C} 8-\mathrm{C} 7-\mathrm{C} 6$ & $-0.8(3)$ & $\mathrm{C} 5-\mathrm{C} 6-\mathrm{C} 1-\mathrm{C} 2$ & $-3.0(3)$ \\
\hline $\mathrm{O} 2-\mathrm{S} 1-\mathrm{N} 1-\mathrm{C} 8$ & $58.34(15)$ & $\mathrm{C} 14-\mathrm{C} 13-\mathrm{C} 12-\mathrm{N} 3$ & $-179.36(19)$ \\
\hline $\mathrm{O} 2-\mathrm{S} 1-\mathrm{N} 1-\mathrm{C} 9$ & $-164.09(14)$ & $\mathrm{C} 9-\mathrm{N} 1-\mathrm{C} 8-\mathrm{C} 10$ & $86.3(2)$ \\
\hline $\mathrm{O} 2-\mathrm{S} 1-\mathrm{C} 1-\mathrm{C} 6$ & $-74.44(16)$ & $\mathrm{C} 9-\mathrm{N} 1-\mathrm{C} 8-\mathrm{C} 7$ & $-96.7(2)$ \\
\hline
\end{tabular}

Symmetry code: (i) $-x+1,-y+2,-z+1$.

Hydrogen-bond geometry $\left(A,{ }^{\circ}\right)$

\begin{tabular}{lllll}
\hline$D-\mathrm{H} \cdots A$ & $D-\mathrm{H}$ & $\mathrm{H} \cdots A$ & $D \cdots A$ & $D-\mathrm{H} \cdots A$ \\
\hline $\mathrm{O} 3-\mathrm{H} 3 \cdots \mathrm{O} 4$ & 0.82 & 1.91 & $2.6221(18)$ & 145 \\
$\mathrm{O} 6-\mathrm{H} 6 \cdots \mathrm{N} 3$ & 0.82 & 1.80 & $2.615(2)$ & 174
\end{tabular}


supporting information

$\mathrm{N} 2-\mathrm{H} 2 \cdots \mathrm{O} 5$

$\mathrm{O} 3-\mathrm{H} 3 \cdots \mathrm{O} 4{ }^{\mathrm{ii}}$

Symmetry code: (ii) $-x+1,-y+1,-z$.
0.86

0.82
2.09

2.51
$2.922(2)$

$2.944(2)$
164

114 